\title{
Increased serum adiponectin levels in type 1 diabetic patients with microvascular complications
}

Received: 21 December 2004 / Accepted: 18 April 2005 / Published online: 3 August 2005

(C) Springer-Verlag 2005

\begin{abstract}
Aims/hypothesis: Low serum adiponectin (ADPN) has been associated with increased risk of cardiovascular disease (CVD) and retinopathy in patients with type 2 diabetes mellitus. In type 1 diabetic patients, the relationship between ADPN and the presence of vascular complications is largely unknown. Methods: We investigated the relationship between serum ADPN and the presence of retinopathy, nephropathy and CVD in patients with type 1 diabetes, divided into matched groups with normoalbuminuria and no retinopathy $(n=67)$, simplex retinopathy $(n=106)$ or proliferative retinopathy $(n=19)$, and nephropathy with simplex $(n=62)$ or proliferative retinopathy $(n=137)$. Healthy control subjects $(n=25)$ were included. Results: Serum ADPN was increased in subjects with type 1 diabetes compared with control subjects $(p<0.0001)$. Further, serum ADPN was higher in patients with than in those without nephropathy $(p<0.0001)$. It was also higher in normoalbuminuric patients with than in those without proliferative retinopathy $(p<0.0001)$. These differences remained significant after adjustment for known risk factors $(p<0.03)$. CVD was also associated with elevated ADPN levels $(p<0.05)$, but this difference became insignificant after risk factor adjustment. The most important predictor of serum ADPN was sex $\left(r^{2}=19 \%\right)$ in normoalbuminuric patients and GFR in patients with nephropathy $\left(r^{2}=18 \%\right)$. Conclusion/interpretation: Patients with type 1 diabetes and microvascular complications have higher serum levels of ADPN than patients without complications. It remains to
\end{abstract}

\footnotetext{
J. Frystyk $(\bowtie) \cdot$ T. Krarup Hansen · A. Flyvbjerg

Medical Research Laboratories, Clinical Institute and Medical Department M (Diabetes and Endocrinology),

Aarhus University Hospital,

Aarhus C, Denmark

e-mail: jan@frystyk.dk

Tel.: +45-89492166

Fax: $+45-89492150$

L. Tarnow $\cdot$ H.-H. Parving

Steno Diabetes Center,

Gentofte, Denmark
}

be clarified whether elevated levels of ADPN are pathogenically related to the development of microvascular complications or represent a beneficial counter-regulatory response.

Keywords Adiponectin - Cardiovascular disease . Gender · GFR · Microvascular complications · Nephropathy - Retinopathy $\cdot$ Type 1 diabetes $\cdot$ Type 1 diabetic patients

Abbreviations ADPN: adiponectin - ADPN-R: adiponectin receptor CVD: cardiovascular disease . ESRF: end-stage renal failure - IHD: ischaemic heart disease - MRS: Minnesota rate scale $\cdot$ NSB: Non-specific binding - UAE: urinary albumin excretion - WHO: World Health Organization

\section{Introduction}

Adiponectin (ADPN) is a polypeptide hormone that was discovered 10 years ago by four independent research groups [1-4]. ADPN is produced exclusively in adipocytes and circulates at very high levels in the bloodstream, where several isoforms have been characterised [5, 6]. Full-length (30 kDa) ADPN undergoes post-translational modification by hydroxylation and glycosylation, resulting in trimers and higher-order polymers. Proteolytic cleavage products containing a globular domain of ADPN have also been detected [7]. Interestingly, the ability of ADPN to polymerise appears to be important for its biological activity $[5,6]$. Recently, two cell surface receptors for ADPN (ADPN$\mathrm{R} 1$ and -R2) were cloned: the receptors were detectable in most tissues, but liver and muscle showed by far the most prominent expression [8]. ADPN-R activation has been shown to stimulate AMP-activated protein kinase and peroxisome proliferator-activated receptor- $\gamma$ ligand activity, fatty-acid oxidation and glucose uptake $[8,9]$, and these actions may explain the suggested role of ADPN as an endogenous insulin sensitiser [10,11].

ADPN has gained considerable interest due to its suggested involvement in cardiovascular disease (CVD) [12]. 
In experimental studies, ADPN has been shown to exert anti-inflammatory and anti-atherosclerotic effects, and to inhibit neointimal thickening and vascular smooth muscle cell proliferation in mechanically injured arteries [13-15]. In humans, two cross-sectional investigations have reported lower levels of ADPN in patients with than without CVD [16, 17], and a recent nested case-control study has shown that high plasma ADPN levels are associated with lower risk of myocardial infarction in men [18]. Thus, there is substantial evidence that low circulating ADPN levels are associated with an increased risk of CVD.

The role of ADPN in the development of microvascular disease (such as diabetic retinopathy and nephropathy) is largely unknown. Experimental studies have reported stimulatory as well as inhibitory effects of ADPN on angiogenesis [12]. Clinically, lean patients with type 2 diabetes suffering from diabetic retinopathy (proliferative as well as non-proliferative) are reported to have lower levels of ADPN than matched patients without retinopathy [19]. On the other hand, lean as well as obese type 2 diabetic patients have normal to subnormal ADPN levels [19-21], whereas patients with type 1 diabetes have supranormal levels [2224]. At the time of writing, there is sparse information on the association between serum ADPN and the presence of micro- and macrovascular complications in type 1 diabetes. Therefore, the present study aimed to investigate the possible relationship between circulating ADPN levels and the presence of retinopathy, nephropathy and CVD in a cross-sectionally collected cohort of patients with type 1 diabetes.

\section{Subjects, materials and methods}

Subjects

The patient population consisted of 391 subjects with longlasting type 1 diabetes recruited from the outpatient clinic at Steno Diabetes Center, Gentofte, Denmark. Data on the diabetic study population have been published previously [25]. In brief, approximately half of the patients $(n=199)$ had diabetic nephropathy, which was defined on the basis of persistent albuminuria (urinary albumin excretion rate [UAE] $>300 \mathrm{mg} / 24 \mathrm{~h}$ in at least two of three consecutive 24-h urine collections), the presence of simplex retinopathy or proliferative retinopathy, and no evidence of other kidney or renal tract disease. The remaining patients $(n=192)$ had stable normoalbuminuria (UAE $<30 \mathrm{mg} / 24 \mathrm{~h}$ ) and were matched for sex, age and duration of diabetes (Table 1).

All patients were examined for ischaemic heart disease (IHD) using a 12-lead ECG, which was subsequently evaluated according to the Minnesota rate scale (MRS) [26] in a blinded fashion by two trained observers. IHD was diagnosed if the ECG showed signs of probable myocardial infarction (MRS 1.1-1.2) or possible myocardial ischaemia (MRS 1.3, 4.1-4.4, 5.1-5.3 or 7.1), or if the patients reported a history of either angina pectoris or myocardial infarction defined in accordance with the Rose and the World Health Organization (WHO) criteria [26]. Positive manifestations of CVD were signs of ischaemic heart disease, as defined above, or a history of stroke or intermittent claudication, when interviewed with the WHO cardiovascular questionnaire [26].

The study also included a small group of healthy controls $(n=25)$, who were recruited from the employees of The University Hospital in Aarhus. This study group was included with only one purpose: to compare levels of ADPN in healthy subjects and patients with type 1 diabetes.

All subjects gave informed written consent to participation. The study was approved by the local ethics committees and carried out in accordance with the Declaration of Helsinki as revised in 2000 (http://www.wma.net/e/policy/b3.htm).

\section{Methods}

All investigations were performed in the morning following an overnight fast. Venous blood was drawn with minimal
Table 1 Clinical characteristics of the two diabetic study groups

Data are means $\pm \mathrm{SD}$, median with interquartile range (IQR) or percentage

GFR was estimated by the Levey formula [27] in patients with normoalbuminuria and measured by the ${ }^{51} \mathrm{Cr}$-EDTA method in patients with nephropathy

${ }^{a}$ ANOVA $p$-value when comparing healthy control subjects, normoalbuminuric patients and patients with nephropathy. Other $p$ values refer to comparisons between the two diabetic groups

\begin{tabular}{llll}
\hline & Normoalbuminuria $(n=192)$ & Nephropathy $(n=199)$ & $p$ value \\
\hline Men/women & $122 / 77$ & $118 / 74$ & $\mathrm{NS}^{\mathrm{a}}$ \\
Age $($ years) & $42.7 \pm 10.2$ & $40.9 \pm 9.6$ & $\mathrm{NS}^{\mathrm{a}}$ \\
BMI $\left(\mathrm{kg} / \mathrm{m}^{2}\right)$ & $23.6 \pm 2.5$ & $24.0 \pm 3.3$ & $\mathrm{NS}^{\mathrm{a}}$ \\
Diabetes duration (years) & $26.8 \pm 8.5$ & $27.7 \pm 7.9$ & $\mathrm{NS}$ \\
Daily insulin dose (units) & $38(32-46)$ & $40(34-50)$ & $<0.05$ \\
HbA c $^{2}$ ) & $8.5 \pm 1.1$ & $9.6 \pm 1.5$ & $<0.0001$ \\
Systolic BP (mmHg) & $132 \pm 18$ & $151 \pm 23$ & $<0.0001$ \\
Diastolic BP (mmHg) & $75 \pm 10$ & $86 \pm 13$ & $<0.0001$ \\
UAE (mg/24 h) & $8(5-13)$ & $796(342-2,079)$ & $<0.0001$ \\
Serum creatinine ( $\mu$ mol/l) & $76(70-83)$ & $103(82-134)$ & $<0.0001$ \\
GFR (ml/min) & $94 \pm 16$ & $74 \pm 34$ & $<0.0001$ \\
Retinopathy (\%) & & & \\
None & 35 & - & \\
Simplex & 55 & 31 & \\
Proliferative & 10 & 69 & \\
\hline
\end{tabular}


stasis from an antecubital vein. Clotted blood was centrifuged within $1 \mathrm{~h}$ and serum stored at $-80^{\circ} \mathrm{C}$. Arterial BP was measured after at least $10 \mathrm{~min}$ of rest in the supine position.

UAE, $\mathrm{HbA}_{1} \mathrm{c}$ and serum creatinine were assayed with routine laboratory methods. In normoalbuminuric patients, GFR was estimated on the basis of serum creatinine using the Levey formula as previously described (estimated GFR) [27]. In patients with nephropathy, GFR was measured after a single injection of radiolabelled EDTA $\left(3.7 \mathrm{MBq}{ }^{51} \mathrm{Cr}-\right.$ EDTA) by determination of radioactivity in venous blood samples collected 180, 200, 220 and $240 \mathrm{~min}$ after the injection (measured GFR) [25].

Serum ADPN was determined by a novel in-house timeresolved immunofluorometric assay based on reagents from R \& D Systems (Abingdon, UK). The assay was performed in 96-well microtitre plates (Nunc, Roskilde, Denmark) coated overnight with monoclonal ADPN antibody MAB 10651 dissolved in phosphate buffer. Next day, plates were washed once and blocked for $2 \mathrm{~h}$ at room temperature with $1 \%(\mathrm{w} / \mathrm{v})$ BSA dissolved in phosphate buffer. After blocking, wells were washed once and $25 \mu \mathrm{l}$ of antigen (standard or unknown samples) was added. Assay standards (recombinant full-length human ADPN) were made by serial dilution in assay buffer $(40 \mathrm{mmol} / 1$ phosphate, $\mathrm{pH} 8.0,0.2 \% \mathrm{w} / \mathrm{v}$ human serum albumin [Behring AG, Marburg, Germany], 0.9 w/v NaCl, $2 \%$ v/v Tween 20 , $1.6 \mathrm{~g} / 1$ Titriplex V, $0.05 \% \mathrm{w} / \mathrm{v}$ SDS and $0.05 \% \mathrm{w} / \mathrm{v} \mathrm{NaN}$ ) and ranged from 2 to $500 \mu \mathrm{g} / \mathrm{l}$. Serum samples were pretreated with SDS: $10 \mu \mathrm{l}$ serum was added to $50 \mu \mathrm{l}$ of assay buffer containing $2 \%(\mathrm{w} / \mathrm{v}) \mathrm{SDS}$, and the samples were incubated for $1 \mathrm{~h}$ at room temperature. After incubation, all serum samples were further diluted in assay buffer without SDS (final dilution 1:206), resulting in a final SDS concentration equivalent to what was present in the buffer used for ADPN standards.

Standards and unknown samples were analysed in duplicate, non-specific binding (NSB) in quadruplicate. Antigens were incubated for $3 \mathrm{~h}$ at room temperature on a plate shaker together with $175 \mu \mathrm{l}$ assay buffer containing a second polyclonal biotinylated ADPN antibody (BAM 1065) and streptavidin-europium (Perkin Elmer Life Sciences, Turku, Finland). After $3 \mathrm{~h}$, all wells were washed six times and Enhancement Solution (Perkin Elmer Life Sciences) was added; they were incubated on a plate shaker and finally measured in a time-resolved fluorometer.

The ADPN standard curve was linear within the interval from 2 to $500 \mu \mathrm{g} / \mathrm{l}$. NSB averaged $\sim 2,000 \mathrm{cps}$, the lowest standard $\sim 4,000 \mathrm{cps}$ and the highest standard $\sim 600,000$ cps. Serum ADPN diluted in parallel with the standard curve (data not shown). The detection limit (NSB plus $3 \mathrm{SD}$ ) was estimated to be less than $1.5 \mu \mathrm{g} / \mathrm{l}$. Within-assay CVs of standards and unknown samples averaged less than 5\%. Between-assay CVs were estimated by repetitive analysis of a control sample diluted 1:2,500, 1:500 and 1:50 respectively. After 48 setups, betweenassay $\mathrm{CVs}$ averaged $12.2 \%$ at $4.8 \mu \mathrm{g} / \mathrm{l}$ (final dilution $1: 2,500$ ), $9.3 \%$ at $23 \mu \mathrm{g} / 1$ (final dilution $1: 500$ ) and $7.9 \%$ at $234 \mu \mathrm{g} / \mathrm{l}$ (final dilution 1:50). The recovery of exogenously added ADPN to serum was $101 \pm 1 \%$ (mean \pm SEM based on ten samples). Repetitive thawing and freezing of serum for up to seven cycles did not alter the immunoreactive levels of ADPN significantly (data not shown).

\section{Statistics}

Serum creatinine concentrations, UAE and daily insulin doses were non-normally distributed and values are given as medians with interquartile ranges. All other values are given as mean \pm SD. Spearman correlation with two-tailed probability values was used to estimate the strength of association between variables. For non-normally distributed variables, comparisons between groups were performed by means of the Mann-Whitney $U$-test or the Kruskal-Wallis test, whereas unpaired Student's $t$-test or ANOVA was used for normally distributed variables. A chi square test was used for comparison between groups of non-continuous variables. Multiple linear regression and forward stepwise regression analyses were used to determine the strongest predictors of circulating ADPN, and a general linear model (GLM) univariate procedure was used to investigate the association of ADPN with retinopathy and nephropathy. Statistical significance was assumed at $p<0.05$. All statistical calculations were performed with SPSS for Windows version 12.0 (SPSS, Chicago, IL, USA).

\section{Results}

Clinical characteristics and mean serum ADPN levels in the three study groups

The two diabetic groups were matched with regard to sex distribution, age, BMI and diabetes duration, whereas insulin dosage, $\mathrm{HbA}_{1} \mathrm{c}$ and $\mathrm{BP}$ (diastolic and systolic) were higher in those with nephropathy (Table 1). The control group had similar mean age (40.1 \pm 10.6 years), BMI (24.0 \pm $3.3 \mathrm{~kg} / \mathrm{m}^{2}$ ) and sex distribution (12 males and 13 females) as the two diabetic groups (Table 1). However, the only purpose of including healthy controls was to confirm that levels of ADPN were increased in type 1 diabetes (controls vs all patients with type 1 diabetes, $13.5 \pm 4.7$ vs. $23.7 \pm$ $14.3 \mathrm{mg} / 1 ; p<0.0001)$ as previously reported [22-24], and therefore no further statistical analysis was performed in this group.

The relationship between serum ADPN and clinical characteristics

Sex differences, with higher ADPN levels in women than men, were observed in both normoalbuminuric patients $(22.4 \pm 8.4$ vs $15.9 \pm 5.0 \mathrm{mg} / \mathrm{l} ; p<0.0001)$ and patients with nephropathy $(32.1 \pm 17.0$ vs $26.3 \pm 17.3 \mathrm{mg} / 1 ; p<0.005)$. Serum ADPN correlated inversely with BMI and daily insulin dose, and positively with age and diabetes duration in both groups (Table 2). Interestingly, neither group showed any relationship between serum ADPN and long- 
Table 2 Univariate linear regression analyses

\begin{tabular}{|c|c|c|}
\hline Serum ADPN vs & $\begin{array}{l}\text { Normoalbuminuria } \\
(n=192)\end{array}$ & $\begin{array}{l}\text { Nephropathy } \\
(n=199)\end{array}$ \\
\hline BMI & $r=-0.21 ; p<0.005$ & $r=-0.28 ; p<0.001$ \\
\hline Age & $r=0.17 ; p<0.02$ & $r=0.20 ; p<0.006$ \\
\hline Diabetes duration & $r=0.24 ; p<0.001$ & $r=0.20 ; p<0.007$ \\
\hline $\begin{array}{l}\text { Daily insulin } \\
\text { dose }\end{array}$ & $r=-0.36 ; p<0.001$ & $r=-0.36 ; p<0.001$ \\
\hline $\mathrm{HbA}_{1} \mathrm{c}$ & NS & NS \\
\hline Systolic BP & NS & $r=0.32 ; p<0.001$ \\
\hline Diastolic BP & NS & NS \\
\hline UAE & NS & $r=0.17 ; p<0.02$ \\
\hline GFR & $r=-0.16 ; p<0.05$ & $r=-0.42 ; p<0.001$ \\
\hline
\end{tabular}

GFR was estimated by the Levey formula [27] in patients with normoalbuminuria and measured by the ${ }^{51} \mathrm{Cr}$-EDTA method in patients with nephropathy

term glycaemic control, as estimated by $\mathrm{HbA}_{1} \mathrm{c}$. Finally, a significant correlation between ADPN and systolic BP was observed in patients with nephropathy.

In the present study, none of the patients was treated with angiotensin II receptor blockers, whereas eight patients with normoalbuminuria and 105 patients with nephropathy were treated with an ACE inhibitor. There was no difference between normoalbuminuric patients with and without ACE inhibitor treatment $(p=0.3)$. In patients with nephropathy, subjects treated with ACE inhibitors tended to have higher levels of ADPN than those without ACE inhibitor treatment $(30.8 \pm 19.2$ vs $26.0 \pm 14.9 \mathrm{mg} / \mathrm{l}$; $p<0.06$ ). However, this borderline significance disappeared after adjustment for diabetes duration, sex, daily insulin dosage, $\mathrm{HbA}_{1} \mathrm{c}, \mathrm{BMI}$ and systolic $\mathrm{BP}(p=0.3)$. When data were also adjusted for differences in UAE and GFR, the $p$-value increased to 0.7 .

\section{Relationship between serum ADPN and kidney function}

Patients with nephropathy had higher ADPN levels than patients with normoalbuminuria $(28.5 \pm 17.4$ vs $18.4 \pm$ $7.2 \mathrm{mg} / 1 ; p<0.0001)$. Interestingly, this difference remained significant after adjustment for known risk factors for nephropathy, as well as after adjustment for differences in GFR and UAE (Table 3).

Serum ADPN correlated inversely with estimated GFR in patients with normoalbuminuria and with measured GFR in patients with nephropathy (Table 2). Serum ADPN correlated positively with UAE, but only in patients with nephropathy (Table 2). The relationship between measured GFR and serum ADPN is further illustrated in Fig. 1, which shows levels in patients with nephropathy subgrouped into GFR tertiles.

Multiple linear and forward stepwise regression analysis, including sex, BMI, daily insulin dose, diabetes duration and estimated GFR, revealed sex to be the most
Table 3 Patients with normoalbuminuria vs. nephropathy

\begin{tabular}{|c|c|c|c|}
\hline \multirow[t]{2}{*}{ Model } & \multicolumn{2}{|c|}{$\begin{array}{l}\text { ADPN level (mg/l) estimated means } \\
(95 \% \mathrm{CI})\end{array}$} & \multirow[t]{2}{*}{$p$-value } \\
\hline & $\begin{array}{l}\text { Normoalbuminuria } \\
(n=191)\end{array}$ & $\begin{array}{l}\text { Nephropathy } \\
(n=199)\end{array}$ & \\
\hline 1. Crude & $18.4(16.5-20.3)$ & $28.5(26.6-30.4)$ & $<0.0001$ \\
\hline $\begin{array}{l}\text { 2. After adjustment } \\
\text { for diabetes } \\
\text { duration, sex, } \\
\text { daily insulin } \\
\text { dose, } \mathrm{HbA}_{1} \mathrm{c} \text {, } \\
\text { BMI and } \\
\text { systolic BP }\end{array}$ & $19.5(17.6-21.4)$ & $27.3(25.5-29.1)$ & $<0.0001$ \\
\hline 3. $2+\mathrm{UAE}$ and GFR & $20.7(18.8-22.6)$ & $26.2(24.4-28.1)$ & 0.0003 \\
\hline
\end{tabular}

important predictor of ADPN levels among patients with normoalbuminuria, accounting for $19 \%$ of the observed variation. Among patients with nephropathy, regression analysis, including sex, diabetes duration, BMI, daily insulin dose, systolic BP, UAE and measured GFR, revealed GFR to be the most important predictor of ADPN levels, accounting for $18 \%$ of the observed variation.

The relationship between serum ADPN and retinopathy

Serum ADPN was significantly higher in normoalbuminuric patients with proliferative retinopathy $(23.8 \pm 11.5 \mathrm{mg} / \mathrm{l})$ than in patients without retinopathy $(17.4 \pm 6.1 \mathrm{mg} / 1 ; p<0.005)$ and simplex retinopathy $(18.2 \pm 6.5 \mathrm{mg} / 1 ; p<0.02)$, whereas no

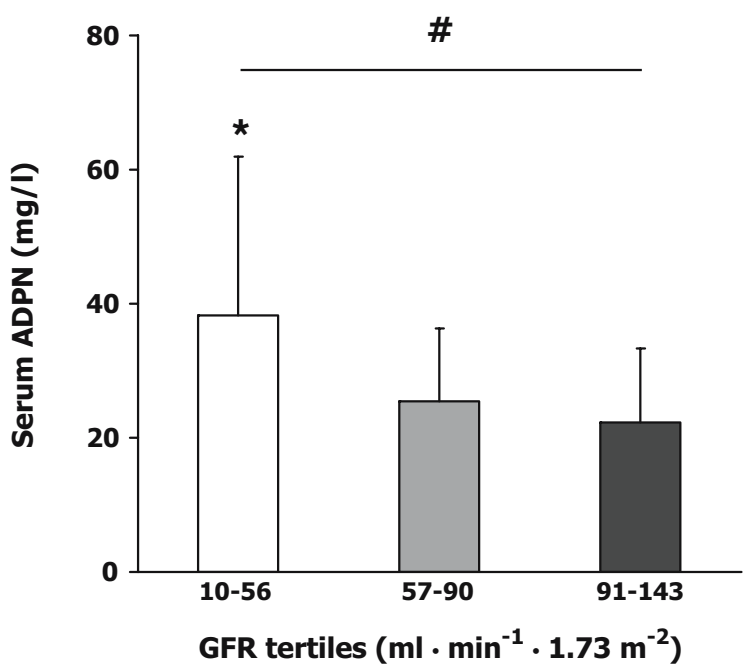

Fig. 1 Unadjusted serum ADPN in diabetic patients with nephropathy $(n=199)$ subgrouped into GFR tertiles. GFR was measured using the ${ }^{51}$ Cr-EDTA method. Data are means and SD. ${ }^{*} p<0.0001$ (ANOVA); ${ }^{*} p<0.0001$, comparing the lower GFR tertile and the two upper tertiles 


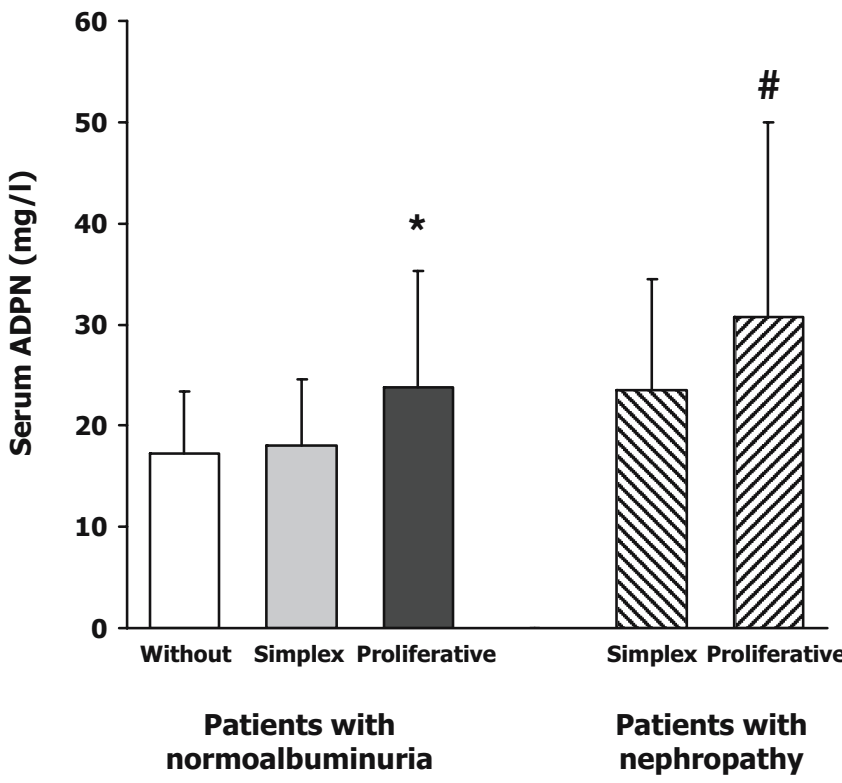

Fig. 2 Unadjusted serum ADPN levels in normoalbuminuric patients without retinopathy (open column), with simplex retinopathy (grey column) and with proliferative retinopathy (black column). Also shown (hatched columns): unadjusted serum ADPN levels in patients with nephropathy plus simplex retinopathy and patients with nephropathy plus proliferative retinopathy. Data are means and SD. ${ }^{*} p<0.05$ compared with normoalbuminuric patients without retinopathy or with simplex retinopathy; ${ }^{*} p<0.05$ compared with patients with nephropathy and simplex retinopathy

difference between the two latter groups was observed (Fig. 2). Of note, the difference in serum ADPN between the three retinopathy groups remained significant after adjustment for known risk factors and differences in renal function in a multivariate analysis (Table 4).

Serum ADPN was also significantly higher among patients with nephropathy and proliferative retinopathy than in those with nephropathy and simplex retinopathy $(30.8 \pm 19.2$ vs $23.5 \pm$ $11.0 \mathrm{mg} / 1 ; p<0.01$ ) (Fig. 2). This difference became borderline significant when adjusting for diabetes duration, sex, daily insulin dose, $\mathrm{HbA}_{1} \mathrm{c}, \mathrm{BMI}$ and systolic $\mathrm{BP}$ (Table 5). However, patients with nephropathy and proliferative retinopathy had significantly higher levels of serum creatinine (106 [88-144] vs 91 [76-119] $\mu \mathrm{mol} / \mathrm{l} ; p<0.005)$ and a lower measured GFR (66 [41-92] vs $89[71-112] \mathrm{ml} \cdot \mathrm{min}^{-1} \cdot 1.73 \mathrm{~m}^{-2}$ $p<0.0001)$ when compared with patients with nephropathy
Table 5 Retinopathy in patients with nephropathy

\begin{tabular}{|c|c|c|c|}
\hline \multirow[t]{2}{*}{ Model } & \multicolumn{2}{|c|}{$\begin{array}{l}\text { ADPN levels }(\mathrm{mg} / \mathrm{l}) \text { estimated } \\
\text { means }(95 \% \mathrm{CI})\end{array}$} & \multirow[t]{2}{*}{$p$-value } \\
\hline & $\begin{array}{l}\text { Simplex } \\
\text { retinopathy } \\
(n=62)\end{array}$ & $\begin{array}{l}\text { Proliferative } \\
\text { retinopathy } \\
(n=137)\end{array}$ & \\
\hline 1. Crude & $23.5(19.2-27.8)$ & $30.8(27.9-33.7)$ & 0.006 \\
\hline $\begin{array}{l}\text { 2. After adjustment } \\
\text { for diabetes } \\
\text { duration, sex, } \\
\text { daily insulin } \\
\text { dose, } \mathrm{HbA}_{1} \mathrm{c}, \mathrm{BMI} \\
\text { and systolic BP }\end{array}$ & $25.3(21.4-29.3)$ & $29.9(27.3-32.5)$ & 0.064 \\
\hline 3. $2+\mathrm{GFR}$ & $26.8(22.8-30.7)$ & $29.4(26.8-32.1)$ & 0.29 \\
\hline 4. 2+UAE and GFR & $27.0(23.1-31.0)$ & $29.3(26.7-32.0)$ & 0.36 \\
\hline
\end{tabular}

and simplex retinopathy, and the difference in serum ADPN became insignificant after adjustment for differences in kidney function (Table 5).

The relationship between serum ADPN and CVD

Twenty-four patients with normoalbuminuria and 60 patients with nephropathy suffered from CVD, and in both groups ADPN levels were higher than in those without CVD (normoalbuminuria, $21.2 \pm 10.0$ vs $18.0 \pm$ $6.7 \mathrm{mg} / \mathrm{l} ; \quad p<0.05$; nephropathy, $32.8 \pm 19.9$ vs $26.7 \pm$ $16.4 \mathrm{mg} / 1 ; p<0.05)$. However, these differences became insignificant after adjustment for differences in diabetes duration, sex, daily insulin dose, $\mathrm{HbA}_{1} \mathrm{c}, \mathrm{BMI}$, systolic $\mathrm{BP}$ and renal function in a multivariate analysis (data not shown).

\section{Discussion}

This cross-sectional study investigated the relationship between serum ADPN levels and the presence of micro- and macrovascular complications in a cohort of approximately 400 type 1 diabetic patients. Our data show that serum ADPN is supranormal in type 1 diabetes irrespective of the presence of complications. Of note, ADPN levels are even higher in patients suffering from microvascular complications. Thus,
Table 4 Retinopathy in patients with normoalbuminuria

\begin{tabular}{|c|c|c|c|c|}
\hline \multirow[t]{2}{*}{ Model } & \multicolumn{3}{|c|}{ ADPN level (mg/l) estimated means $(95 \% \mathrm{CI})$} & \multirow[t]{2}{*}{$p$-value } \\
\hline & $\begin{array}{l}\text { No } \\
\text { retinopathy } \\
(n=67)\end{array}$ & $\begin{array}{l}\text { Simplex } \\
\text { retinopathy } \\
(n=106)\end{array}$ & $\begin{array}{l}\text { Proliferative } \\
\text { retinopathy } \\
(n=19)\end{array}$ & \\
\hline 1. Crude & $17.4(15.6-19.0)$ & $18.2(16.8-19.5)$ & $23.8(20.6-27.0)$ & 0.002 \\
\hline $\begin{array}{l}\text { 2. After adjustment for } \\
\text { diabetes duration, } \\
\text { sex, daily insulin dose, } \\
\mathrm{HbA}_{1} \mathrm{c}, \mathrm{BMI} \text { and systolic BP }\end{array}$ & $17.2(15.7-18.6)$ & $18.4(17.3-19.6)$ & $21.6(18.7-24.6)$ & 0.026 \\
\hline 3. $2+\mathrm{UAE}$ and GFR & $17.3(15.8-18.7)$ & $18.4(17.3-19.6)$ & $21.7(18.7-24.7)$ & 0.032 \\
\hline
\end{tabular}


patients with nephropathy had higher levels of ADPN than patients with normoalbuminuria, and this difference remained significant after adjustment for known risk factors, including differences in UAE and GFR. In normoalbuminuric patients, the presence of proliferative retinopathy was associated with higher levels of ADPN, compared with matched counterparts with either no or simplex retinopathy, and again this difference remained significant after adjustment for known risk factors. Levels of ADPN were similar in patients with and without CVD when differences in known risk factors were taken into account.

Serum ADPN was elevated in all diabetic subgroups, irrespective of the presence of complications, pointing to an effect of type 1 diabetes per se on serum ADPN. However, the underlying mechanism is unknown and may involve factors that appear to control ADPN synthesis/ secretion (insulin, TNF- $\alpha$, IL-6, glucocorticoids and $\beta$ adrenergic agonists) [28] as well as alterations in ADPN clearance. In this context it is interesting that serum ADPN was positively related to diabetes duration in patients with normoalbuminuria as well as nephropathy. This finding is in accordance with observations that we made recently in another cohort of type 1 diabetic patients (Lindström et al., unpublished results). In that study it was possible to divide patients into two highly matched groups of C-peptidepositive and -negative subjects, and it appeared that serum ADPN was normal in C-peptide-positive patients but elevated by $70 \%$ in C-peptide-negative patients, despite a similar $\mathrm{HbA}_{1} \mathrm{c}$ and body composition. However, diabetes duration was almost three times longer in C-peptide-negative patients. Taking these results together with the present study, it may therefore be speculated that the underlying mechanism that is responsible for the elevated serum ADPN levels in long-term type 1 diabetic patients is related to diabetes duration and/or residual beta cell function. However, this hypothesis deserves further investigation.

The metabolic degradation and clearance of ADPN is largely unknown. However, renal involvement appears likely. Non-diabetic patients with end-stage renal failure (ESRF) have approximately a two-fold elevated serum level [29], which decreases towards control values after successful kidney transplantation [30]. Furthermore, a relationship between serum ADPN and kidney function, as estimated by serum creatinine or creatinine clearance, has been observed previously in women with dyslipidaemia [31], subjects with hypertension [32] and patients with type 2 diabetes [21], and as shown here this relationship also appears to be valid in patients with type 1 diabetes. Interestingly, in a large cohort of Pima Indians with type 2 diabetes, ADPN levels increased progressively with elevated UAE and serum creatinine [21]. Although a direct comparison was not performed, levels of ADPN appeared to be at least numerically twice as high in type 2 diabetes patients with macroalbuminuria (albumin:creatinine ratio $>$ 300) and serum creatinine levels above $110 \mu \mathrm{mol} / \mathrm{l}$, compared with healthy controls (geometric ADPN means, 11 vs $5.6 \mathrm{mg} / \mathrm{l})$. This observation is in agreement with our data, and therefore it may be concluded that deteriorated kidney function increases serum ADPN levels in both type 1 diabetes and type 2 diabetes [21] as well as in ESRF [29]; accordingly, differences in kidney function need to be taken into consideration when comparing serum ADPN levels in various study groups. On the other hand, even after adjustment for differences in kidney function, serum ADPN remained elevated in patients with nephropathy compared with normoalbuminuric patients (Table 3 ). The study groups were also examined for differences in treatment with ACE inhibitors, because these drugs have been shown to increase serum ADPN in non-diabetic patients with essential hypertension [33], but as shown this did not explain our observations. Thus, there is evidence that mechanisms other than kidney function may be responsible for the higher serum ADPN levels in type 1 diabetic patients with nephropathy.

Proliferative retinopathy was associated with elevated ADPN levels in normoalbuminuric patients. However, we acknowledge that the number of normoalbuminuric patients with proliferative retinopathy was relatively small (19 out of 192 patients), and therefore this finding needs to be confirmed. Of note, a recent cross-sectional study in subjects with nearly normal weight and with sulphonylurea-treated type 2 diabetes reported a stepwise reduction in serum ADPN with increasing severity of retinopathy [19]. This contrasts with our findings in type 1 diabetes. However, in that study no information on GFR or serum creatinine was given; since kidney function also affects ADPN levels in patients with type 2 diabetes [21, 34], this could have biased results. Nevertheless, at present there is no explanation for the apparent discrepancy concerning the association between ADPN and the presence of retinopathy in type 1 and type 2 diabetes.

The diagnosis of CVD was associated with significantly elevated ADPN levels in patients with normoalbuminuria as well as nephropathy. However, after adjustment for known risk factors, these differences were no longer significant, and accordingly, serum ADPN did not appear to be an independent predictor of an increased risk of CVD in type 1 diabetes. However, it should be stressed that the diagnosis of CVD was based on medical history and resting ECG, and so it cannot be ruled out that the use of more advanced diagnostic tools would have yielded a different result.

Many experimental as well as clinical studies indicate that low ADPN levels are harmful and associated with an increased risk of insulin resistance, type 2 diabetes and CVD [17, 18, 35-37]. Conversely, an increment in plasma ADPN, obtained by direct administration, by stimulation with thiazolidinediones or by genetic manipulation, appears to have beneficial effects on insulin sensitivity and the occurrence of atherosclerosis $[10,11,14,38,39]$. It may therefore appear paradoxically that patients with type 1 diabetes or ESRF, who are at increased risk of CVD [40, 41], have supranormal levels of ADPN. One way to interpret the present findings is to conclude that high ADPN levels may be pathogenically related to the development of microvascular complications. However, the finding can 
also be interpreted in the opposite way, as elevated ADPN levels could represent a beneficial compensatory mechanism. Several markers of inflammation are increased in type 1 diabetes, suggesting that it is a state of chronic lowgrade inflammation $[42,43]$. Having the anti-inflammatory and anti-atherosclerotic properties of ADPN in mind, one could hypothesise that increased ADPN levels serve to protect patients at high risk of CVD against the harmful actions of proinflammatory and atherosclerotic agents. In this context it is worth remembering that serum ADPN levels appear to be partly genetically determined [44], and that there seems to be a link between certain single-nucleotide polymorphisms of the ADPN gene and the risk of type 2 diabetes [44-46]. However, to the best of our knowledge there are no data on the possible relationship between ADPN gene polymorphisms and the risk of microvascular complications in type 1 diabetes.

In the present study we have determined total ADPN levels. However, ADPN circulates as various isoforms and polymers, which may differ in receptor affinities as well as metabolic effects [5-7]. To date, virtually all studies have assayed the total concentration of ADPN in either serum or plasma without differentiating between the various isoforms. However, recent data indicate that it is the plasma fraction of high molecular-weight polymers rather than the total concentration of ADPN that is associated with changes in insulin sensitivity in $d b / d b$ mice as well as in type 2 diabetic patients treated with thiazolidinedione [5]. Therefore, future studies concerning the different circulating isoforms (and the high molecular-weight ADPN isoform in particular) may help shed light on the paradoxically elevated ADPN levels in type 1 diabetes (and ESRF).

Women have higher circulating ADPN concentrations than men, presumably as a result of higher plasma levels of the high molecular-weight isoform [6, 36]. As recently shown, the sex difference in ADPN develops during puberty and it is inversely associated with serum levels of androgen, which is believed to suppress ADPN synthesis [47]. In the present study the sex difference was preserved in normoalbuminuric patients, whereas it was absent in patients with nephropathy. Patients with nephropathy are at very high risk of CVD, and, in contrast to the general population, the risk is similar in diabetic men and women. Thus, it could be hypothesised that in patients with nephropathy there is an association between the lack of a sex difference in plasma ADPN and the absence of a female advantage when it comes to the development of CVD [40].

In conclusion, patients with type 1 diabetes and microvascular complications have higher serum ADPN levels than matched counterparts without complications. Thus, serum ADPN was higher in normoalbuminuric patients with than without proliferative retinopathy, and higher levels were also noted in patients with nephropathy compared with patients with normoalbuminuria. Of note, these differences remained significant after adjustment for known risk factors, including GFR. Future studies are required to discover whether ADPN is involved in the pathogenesis of diabetic angiopathy.
Acknowledgements This study was supported by grants from The Danish Health Research Council (grant 22020141), The Danish Diabetes Association, the Institute of Experimental Clinical Research, the University of Aarhus, The Hørslev Foundation, Novo Nordisk Foundation and the Family Hede Nielsen Foundation. The authors wish to thank Lisbeth Jensen, Annette Hansen and Hanne Pedersen for expert technical assistance.

\section{References}

1. Maeda K, Okubo K, Shimomura I, Funahashi T, Matsuzawa Y, Matsubara K (1996) cDNA cloning and expression of a novel adipose specific collagen-like factor, apM1 (AdiPose Most abundant Gene transcript 1). Biochem Biophys Res Commun 221:286-289

2. Scherer PE, Williams S, Fogliano M, Baldini G, Lodish HF (1995) A novel serum protein similar to C1q, produced exclusively in adipocytes. J Biol Chem 270:26746-26749

3. Hu E, Liang P, Spiegelman BM (1996) AdipoQ is a novel adipose-specific gene dysregulated in obesity. J Biol Chem 271:10697-10703

4. Nakano Y, Tobe T, Choi-Miura NH, Mazda T, Tomita M (1996) Isolation and characterization of GBP28, a novel gelatin-binding protein purified from human plasma. J Biochem (Tokyo) 120:803-812

5. Pajvani UB, Hawkins M, Combs TP et al (2004) Complex distribution, not absolute amount of adiponectin, correlates with thiazolidinedione-mediated improvement in insulin sensitivity. J Biol Chem 279:12152-12162

6. Waki H, Yamauchi T, Kamon J et al (2003) Impaired multimerization of human adiponectin mutants associated with diabetes. Molecular structure and multimer formation of adiponectin. J Biol Chem 278:40352-40363

7. Kershaw EE, Flier JS (2004) Adipose tissue as an endocrine organ. J Clin Endocrinol Metab 89:2548-2556

8. Yamauchi T, Kamon J, Ito Y et al (2003) Cloning of adiponectin receptors that mediate antidiabetic metabolic effects. Nature 423:762-769

9. Yamauchi T, Kamon J, Minokoshi Y et al (2002) Adiponectin stimulates glucose utilization and fatty-acid oxidation by activating AMP-activated protein kinase. Nat Med 8:12881295

10. Yamauchi T, Kamon J, Waki H et al (2001) The fat-derived hormone adiponectin reverses insulin resistance associated with both lipoatrophy and obesity. Nat Med 7:941-946

11. Berg AH, Combs TP, Du X, Brownlee M, Scherer PE (2001) The adipocyte-secreted protein Acrp30 enhances hepatic insulin action. Nat Med 7:947-953

12. Goldstein BJ, Scalia R (2004) Adiponectin: a novel adipokine linking adipocytes and vascular function. J Clin Endocrinol Metab 89:2563-2568

13. Kubota N, Terauchi Y, Yamauchi T et al (2002) Disruption of adiponectin causes insulin resistance and neointimal formation. J Biol Chem 277:25863-25866

14. Yamauchi T, Kamon J, Waki H et al (2003) Globular adiponectin protected $o b / o b$ mice from diabetes and ApoE-deficient mice from atherosclerosis. J Biol Chem 278:2461-2468

15. Matsuda M, Shimomura I, Sata M et al (2002) Role of adiponectin in preventing vascular stenosis. The missing link of adipo-vascular axis. J Biol Chem 277:37487-37491

16. Hotta K, Funahashi T, Arita Y et al (2000) Plasma concentrations of a novel, adipose-specific protein, adiponectin, in type 2 diabetic patients. Arterioscler Thromb Vasc Biol 20:1595-1599

17. Kumada M, Kihara S, Sumitsuji S et al (2003) Association of hypoadiponectinemia with coronary artery disease in men. Arterioscler Thromb Vasc Biol 23:85-89

18. Pischon T, Girman CJ, Hotamisligil GS, Rifai N, Hu FB, Rimm EB (2004) Plasma adiponectin levels and risk of myocardial infarction in men. JAMA 291:1730-1737 
19. Yilmaz MI, Sonmez A, Acikel C et al (2004) Adiponectin may play a part in the pathogenesis of diabetic retinopathy. Eur $\mathrm{J}$ Endocrinol 151:135-140

20. Weyer C, Funahashi T, Tanaka S et al (2001) Hypoadiponectinemia in obesity and type 2 diabetes: close association with insulin resistance and hyperinsulinemia. J Clin Endocrinol Metab 86:1930-1935

21. Looker HC, Krakoff J, Funahashi T et al (2004) Adiponectin concentrations are influenced by renal function and diabetes duration in Pima Indians with type 2 diabetes. J Clin Endocrinol Metab 89:4010-4017

22. Mannucci E, Ognibene A, Cremasco F et al (2003) Plasma adiponectin and hyperglycaemia in diabetic patients. Clin Chem Lab Med 41:1131-1135

23. Imagawa A, Funahashi T, Nakamura T et al (2002) Elevated serum concentration of adipose-derived factor, adiponectin, in patients with type 1 diabetes. Diabetes Care 25:1665-1666

24. Perseghin G, Lattuada G, Danna M et al (2003) Insulin resistance, intramyocellular lipid content, and plasma adiponectin in patients with type 1 diabetes. Am J Physiol Endocrinol Metab 285:E1174-E1181

25. Tarnow L, Cambien F, Rossing $P$ et al (1995) Lack of relationship between an insertion/deletion polymorphism in the angiotensin I-converting enzyme gene and diabetic nephropathy and proliferative retinopathy in IDDM patients. Diabetes 44:489-494

26. Rose GA, Blackburn H (1968) Cardiovascular survey methods. Monogr Ser World Health Organ 56:1-188

27. Levey AS, Bosch JP, Lewis JB, Greene T, Rogers N, Roth D (1999) A more accurate method to estimate glomerular fil tration rate from serum creatinine: a new prediction equation. Ann Intern Med 130:461-470

28. Fasshauer M, Paschke R (2003) Regulation of adipocytokines and insulin resistance. Diabetologia 46:1594-1603

29. Zoccali C, Mallamaci F, Tripepi G et al (2002) Adiponectin, metabolic risk factors, and cardiovascular events among patients with end-stage renal disease. J Am Soc Nephrol 13:134-141

30. Chudek J, Adamczak M, Karkoszka H et al (2003) Plasma adiponectin concentration before and after successful kidney transplantation. Transplant Proc 35:2186-2189

31. Matsubara M, Maruoka S, Katayose S (2002) Decreased plasma adiponectin concentrations in women with dyslipidemia. J Clin Endocrinol Metab 87:2764-2769

32. Mallamaci F, Zoccali C, Cuzzola F et al (2002) Adiponectin in essential hypertension. J Nephrol 15:507-511

33. Furuhashi M, Ura N, Higashiura K et al (2003) Blockade of the renin-angiotensin system increases adiponectin concentrations in patients with essential hypertension. Hypertension 42:76-81

34. Schaffler A, Herfarth H, Paul G et al (2004) Identification of influencing variables on adiponectin serum levels in diabetes mellitus type 1 and type 2. Exp Clin Endocrinol Diabetes $112: 383-389$
35. Lindsay RS, Funahashi T, Hanson RL et al (2002) Adiponectin and development of type 2 diabetes in the Pima Indian population. Lancet 360:57-58

36. Spranger J, Kroke A, Mohlig M et al (2003) Adiponectin and protection against type 2 diabetes mellitus. Lancet 361:226-228

37. Daimon M, Oizumi T, Saitoh T et al (2003) Decreased serum levels of adiponectin are a risk factor for the progression to type 2 diabetes in the Japanese population: the Funagata study. Diabetes Care 26:2015-2020

38. Diez JJ, Iglesias P (2003) The role of the novel adipocytederived hormone adiponectin in human disease. Eur J Endocrinol 148:293-300

39. Yu JG, Javorschi S, Hevener AL et al (2002) The effect of thiazolidinediones on plasma adiponectin levels in normal, obese, and type 2 diabetic subjects. Diabetes 51:2968-2974

40. Tuomilehto J, Borch-Johnsen K, Molarius A et al (1998) Incidence of cardiovascular disease in Type 1 (insulin-dependent) diabetic subjects with and without diabetic nephropathy in Finland. Diabetologia 41:784-790

41. Sarnak MJ, Levey AS, Schoolwerth AC et al (2003) Kidney disease as a risk factor for development of cardiovascular disease: a statement from the American Heart Association Councils on Kidney in Cardiovascular Disease, High Blood Pressure Research, Clinical Cardiology, and Epidemiology and Prevention. Hypertension 42:1050-1065

42. Hansen TK, Tarnow L, Thiel S et al (2004) Association between mannose-binding lectin and vascular complications in type 1 diabetes. Diabetes 53:1570-1576

43. Schalkwijk CG, Poland DC, van Dijk W et al (1999) Plasma concentration of C-reactive protein is increased in type I diabetic patients without clinical macroangiopathy and correlates with markers of endothelial dysfunction: evidence for chronic inflammation. Diabetologia 42:351-357

44. Vasseur F, Lepretre F, Lacquemant C, Froguel P (2003) The genetics of adiponectin. Curr Diabetes Rep 3:151-158

45. Fumeron F, Aubert R, Siddiq A et al (2004) Adiponectin gene polymorphisms and adiponectin levels are independently associated with the development of hyperglycemia during a 3-year period: the epidemiologic data on the insulin resistance syndrome prospective study. Diabetes 53:1150-1157

46. Hara K, Boutin P, Mori Y et al (2002) Genetic variation in the gene encoding adiponectin is associated with an increased risk of type 2 diabetes in the Japanese population. Diabetes 51:536540

47. Bottner A, Kratzsch J, Muller G et al (2004) Gender differences of adiponectin levels develop during the progression of puberty and are related to serum androgen levels. J Clin Endocrinol Metab 89:4053-4061 